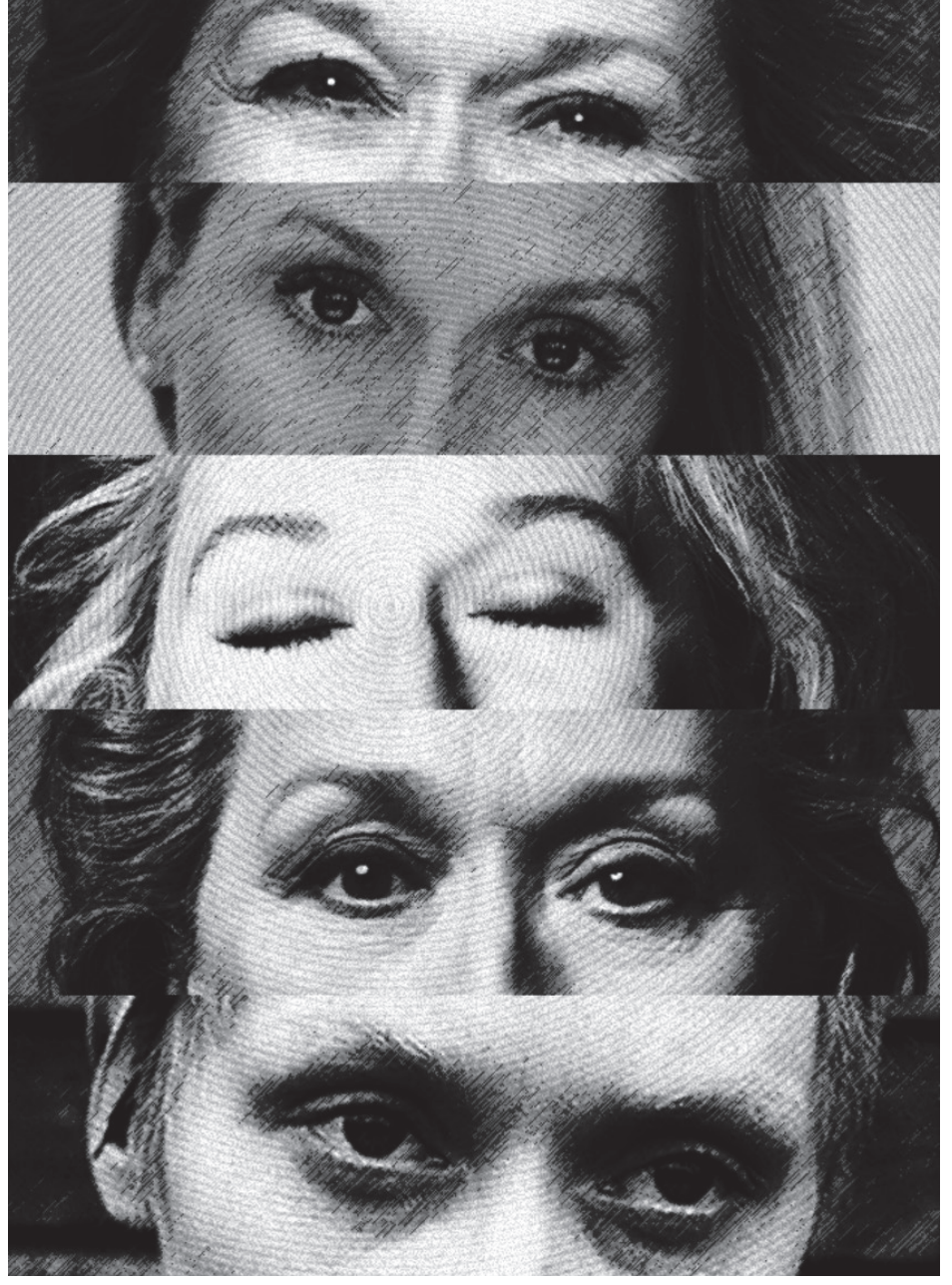

\title{
MERYL STREEP
}

\section{LA ACTRIZ DE LAS MIL VOCES (Y POLIFONÍAS)}

MERYL STREEP, THE ACTRESS OF A

THOUSAND VOICES (AND POLYPHONIES)

Por

Prof.Trad. Guillermo Badenes ${ }^{1}$

Facultad de Lenguas, Universidad Nacional de Córdoba guillermobadenes@fl.unc.edu.ar 
Resumen: El análisis del discurso se presenta como una disciplina de utilidad para los estudiosos de la cultura y un complemento que avanza más allá de los niveles sintácticos, semánticos, morfológicos o fonológicos, para analizar la lengua en su contexto discursivo. Un aspecto de estudio de interés es el discurso referido. Meryl Streep es una actriz reconocida por su capacidad de imitar acentos y dialectos. Cuando recibió un galardón durante la ceremonia de los Globos de Oro de 2017, la actriz construyó su alocución a partir de las palabras (y actos) de otros: el análisis de su discurso de aceptación permite observar cómo los diversos discursos referidos que utilizó consolidan el sentimiento de comunidad y denuncian las políticas del presidente Donald Trump. El presente estudio analiza el discurso de Streep en dicha ocasión al subrayar que las expresiones de un sujeto muchas veces contienen capas de sentido provenientes de otros discursos.

Palabras clave: análisis del discurso, discurso referido, Globos de Oro, Meryl Streep.

Abstract: Discourse analysis is discipline of use for culture scholars, and a complement to go beyond the syntactic, semantic, morphologic or phonologic levels to analyze language in its discursive context. An aspect of study of interest is reported speech. Meryl Streep is a renowned actress famous for her capacity to imitate accents and dialects. When she was given an award at the Golden Globe ceremony in 2017, the actress built her acceptance speech from the words (and actions) or others: a discourse analysis of her address allows us to observe the way in which the reported speech she used makes up a sense of community with her peers and denounces President Donald Trump. This study analyzes Streep's speech on that occasion underlining the fact that the expressions that a person uses often contain layers of meaning coming from the words of others.

Key words:discourse analysis, reported speech, Golden Globes, Meryl Streep.

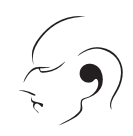

\section{Introducción}

La noche del domingo 8 de enero de 2017, se llevó a cabo la ceremonia anual de entrega de los premios Globo de Oro que imparte en Estados Unidos la Asociación de la Prensa Extranjera de Hollywood, tanto al cine como a la televisión. Los premios, que se otorgan desde 1944, se consideran por su prestigio una antesala de los premios Oscar de la Academia de las Artes y las Ciencias Cinematográficas 
de Estados Unidos. Como todo premio, suscita la atención del público debido a la incertidumbre que genera saber quiénes serán beneficiados con la estatuilla. Sin embargo, desde 1950 existe en la ceremonia de los Globos de Oro una categoría honorífica llamada Premio Cecil B. DeMille, que se adjudica todos los años a un actor, productor o director, en reconocimiento a la trayectoria del galardonado. En la $74^{\circ}$ entrega de premios había solo un nombre seguro que regresaría a casa con una distinción: la actriz Meryl Streep, destinataria de ese galardón por su corpus actoral.

Meryl Streep ha sido siempre una actriz dúctil reconocida en todo el mundo por el don que posee de recrear los más disímiles personajes, pero en especial por su habilidad innata de imitar los acentos más diversos en la lengua inglesa. Esta capacidad le ha valido el mote de «la actriz de las mil voces». Sin embargo, cuando el 8 de enero subió a recibir el Premio Cecil B. DeMille, sorprendió al mundo al convertir su discurso de agradecimiento en un manifiesto político sobre la realidad que atravesaba Estados Unidos ante la inminente asunción de Donald Trump como presidente del país. Tan contundentes fueron sus apreciaciones que le valieron la crítica del propio presidente electo, quien la llamó «sobrevalorada» (citado en Izardi y Wand, 2017, mi traducción). Más allá de cualquier apreciación sobre la capacidad actoral de Streep, resulta interesante llevar a cabo un análisis discursivo de sus palabras, lo que puede dar cuenta de que la «actriz de las mil voces» desplegó en su alocución diversas instancias de polifonía que revelan su posicionamiento político actual.

El análisis del discurso se presenta como una disciplina de utilidad para los estudiosos de la lengua y la cultura, y un complemento que avanza más allá de los niveles sintácticos, semánticos, morfológicos o fonológicos para analizar la lengua en su contexto discursivo. Un aspecto de estudio de particular interés es el del discurso referido, ya que el fluir del habla a menudo presenta ecos, referencias o citas de las palabras de otros. El presente estudio analizará el discurso de Streep al recibir el Premio Cecil B. DeMille, al subrayar que las expresiones de un sujeto muchas veces contienen capas de sentido provenientes de otros discursos, de los que se debe ser consciente en todo momento para comprender los valores culturales inmanentes.

\section{Un discurso, muchas voces}

La actriz organizó su discurso en al menos siete partes: su agradecimiento inicial y una aclaración sobre su disfonía, una referencia a la comunidad de Hollywood, una consideración sobre los extranjeros, una anécdota que la afectó recientemente, ciertas apreciaciones sobre la violencia verbal, una observación sobre la prensa y una reflexión final sobre el papel del actor en la sociedad. En términos de Bajtín, su discurso contó «con la presencia de ciertos enunciados anteriores, suyos y ajenos, con los cuales su enunciado [estableció] toda suerte de relaciones» (García y Tordesillas, 2001, p. 155), lo que le dio al texto distintas instancias de intertextualidad y una arquitectura polifónica que será el objeto de este estudio. 
Tras los agradecimientos iniciales a la Asociación de la Prensa Extranjera de Hollywood, Streep se disculpó por tener la voz ronca, «I've lost my voice in screaming and lamentation this weekend. And I have lost my mind sometime earlier this year» ${ }^{2}$ (Izadi y Wand, 2017). La enunciación de Streep como locutora del acto conlleva indicaciones causales. Estas causas tienen como implicatura la gravedad de la situación política actual del país que la afectó de tal modo que la hizo perder la voz.

A continuación, Streep introdujo un nuevo locutor al orientar su discurso hacia las palabras del actor Hugh Laurie, que ya había recibido un premio esa noche. Streep expresó, «Just to pick up on what Hugh Laurie said, you and all of us in this room, really, belong to the most vilified segments in American society right now. Think about it: 'Hollywood, foreigners and the press'»' (Izadi y Wand, 2017). Se reconoce aquí una instancia mixta de discurso indirecto y de discurso indirecto libre: si bien se cita al locutor, antes de hacerlo, Streep reinterpreta su enunciado. En sus palabras de agradecimiento, Laurie había expresado:

Thank you to the Hollywood Foreign Press Association for this amazing honor. I suppose it's made more amazing by the fact that I'll be able to say I won this at the last-ever Golden Globes [... I I don't mean to be gloomy. It's just that it has the words «Hollywood,» «foreign» and «press» in the title. I also think that to some Republicans, even the word «association» is slightly sketchy ${ }^{4}$ (THR Staff, 2017).

Como he indicado, entiendo que se trata de una instancia mixta de discurso indirecto y de discurso indirecto libre, pues como explican García Negroni y Tordesillas Colado (2001), el discurso indirecto representa «una síntesis, una expansión, una modificación de las palabras con respecto a lo dicho posible, siempre y cuando se atienda al propósito del discurso del locutor citado» (p. 166). Las palabras de Laurie en referencia a Hollywood, la prensa y los extranjeros, apuntan a la ironía de que el nombre de la institución que otorga los premios consiste de tres grupos sobre los que Donald Trump ha expresado reparos. Por su parte, en el discurso indirecto libre de Streep se suma la noción de que estos grupos son denigrados por el presidente electo. La alocución de la actriz se encuentra orientada hacia el enunciado de Laurie, y - a diferencia de lo que indican García Negroni y Tordesillas Colado, «La palabra ajena puede ser utilizada con la misma intención original: se encuadrarían entonces los fenómenos de estilización, de 'skaz', de relato oral puro orientado hacia el discurso ajeno» (2001, p. 161) - no se trata aquí solo de una estilización o de que Streep se convierta en «portadora parcial» de las opiniones de Laurie, sino de que Streep reformula su enunciación para, a través del discurso indirecto libre, hacerlo locutor de un concepto mayor a lo enunciado oportunamente. 
Vale asimismo subrayar que en este discurso indirecto libre, donde Streep convierte a Laurie en enunciador de la apreciación, «you and all of us in this room, really, belong to the most vilified segments in American society right now» ${ }^{5}$ (citado en Izardi y Wand, 2017), Streep construye la primera instancia de comunidad en su enunciación. Como explica Ducrot, «la mayoría (por lo menos) de las lenguas utilizan marcas especiales para designar la función de alocutario» (1994, p. 137). Con el «you» de la segunda persona singular del comienzo - como he indicado - la actriz asegura a Laurie como el enunciador de la idea, aunque en ese momento sea el alocutario (junto con el resto del público presente) del enunciado por encontrarse en la platea. A su vez, de inmediato, la actriz agrega la idea de «all of us in this room, really» (citado en Izardi y Wand, 2017) y con el uso del modificador sobrerrealizante elativo «really», implica a todos y cada uno de los presentes (ella incluida) dentro del grupo vilipendiado en la actualidad: en el mismo acto pone en suspenso el discurso referido de Laurie y se vuelve enunciadora a la vez que locutora de la enunciación, para luego retomar el discurso indirecto libre del actor. Esta instancia inclusiva en el discurso de Streep se repetirá en toda su alocución y esto resulta importante ya que construye un sentido de pertenencia y de comunidad en el grupo: una condición necesaria en su argumentación para contrarrestar los embates de la realidad actual.

A continuación, el locutor, identificado aquí con Streep, refuerza esta idea inclusiva de comunidad con el uso de la primera persona del plural y plantea dos preguntas retóricas que luego responde, «But who are we, and what is Hollywood, anyway? It's just a bunch of people from other places» ${ }^{6}$ (citado en Izadi y Wand, 2017). En las preguntas en sí, como locutora, Streep se ubica tanto en posición de L como de $\lambda$, un personaje que puede, como explican García Negroni y Tordesillas Colado (2001), poseer propiedades «ajenas a sí misma» (p. 177), es decir, en este caso convertirse en parte de la comunidad de Hollywood bajo los ataques de Trump. La respuesta a sus preguntas retóricas solo la ubican en el rol de $\mathrm{L}$, «responsable de la enunciación y considerado únicamente como detentor de esa propiedad» (García y Tordesillas, p. 177). Asimismo, las dos preguntas retóricas contienen un enunciador general -la comunidad de Hollywood en su conjunto-, mientras que en la respuesta Streep se presenta como locutora responsable del punto de vista introducido: la actriz de mayor trayectoria en Hollywood, que ha trabajado con un gran número de los presentes, es la que responde y conoce en profundidad a los diversos actores en el medio. Esta afirmación puede comprobarse por el hecho de que, a continuación, Streep nombra a nueve colegas y detalla sus lugares de nacimiento.

A través de este extenso catálogo de colegas de orígenes diversos, Streep puede volver a atacar la figura de Donald Trump sin mencionarlo. Tras nombrar a las actrices Amy Adams y Natalie Portman, nacidas en Italia e Israel respectivamente, Streep ajusta el tono de voz y se pregunta, «where are their birth certificates?» ${ }^{7}$ (citado en Izadi y Wand, 2017). El cambio prosódico de Streep invierte en este caso la orientación del argumento y, de este modo, puede darle a la pregunta un punto de vista alusivo. Vale recordar en este punto que en 2011, cuando evaluaba la posibilidad de ser 
candidato presidencial, Trump lanzó una campaña en contra de Barack Obama en la que le exigía que mostrara su partida de nacimiento (Keneally, 2015) aduciendo que en realidad Obama no había nacido en Estados Unidos. Meryl Streep realiza una referencia indirecta al discurso Trump al tomar sus palabras «con intención opuesta a la original» (García y Tordesillas, 2001, p. 161). En tal sentido, la actriz utiliza un tono admonitorio para hacer suyas las ideas populistas del presidente electo en cuanto a rechazar a los extranjeros en Estados Unidos.

Como indica Ducrot (1994) tomando la tesis de Sperber y Wilson, «Para que nazca la ironía es preciso que desaparezca toda marca de transmisión, hace falta 'hacer como si' este discurso fuera realmente pronunciado en la enunciación misma» (p. 262). Con el cambio en el tono de su voz, la pregunta se vuelve una parodia que la actriz concluye con una advertencia, «So Hollywood is crawling with outsiders and foreigners. And if we kick them all out, you'll have nothing to watch but football and mixed martial arts, which are not the $\operatorname{arts}^{8}{ }^{8}$ (citado en Izadi y Wand, 2017). Según las ideas de Ducrot,

Hablar de manera irónica equivale, para el locutor L, a presentar la enunciación como el cumplimiento, por parte de E, de actos (preguntas, afirmaciones, promesas, exclamaciones, etc.) absurdos; al mismo tiempo L marca que no asume la responsabilidad de estos actos (1994, p. 262).

Esta negación de la responsabilidad de lo que ocurriría si se retira a los extranjeros de Hollywood -y Estados Unidos-, se ve reflejada en la conclusión final de que, de hacerse, solo se podría ver deportes en televisión. Esta proposición implícita de expresión es lo que vuelve el discurso de la actriz paródico.

Streep continúa sus referencias a los dichos de Donald Trump en el siguiente segmento, la cuarta parte de su discurso. En él, la actriz usa, en términos de Bajtín, la variante activa de orientación hacia el discurso ajeno. Así, «la palabra queda fuera del discurso del autor, pero éste la toma en cuenta y se refiere a ella. El discurso de los otros no aparece, pero determina el enunciado portador» (García y Tordesillas, 2001, p. 161) cuando Meryl Streep le recuerda al público, «It was that moment when the person asking to sit in the most respected seat in our country imitated a disabled reporter» ${ }^{9}$ (citado en Izardi y Wand, 2017).

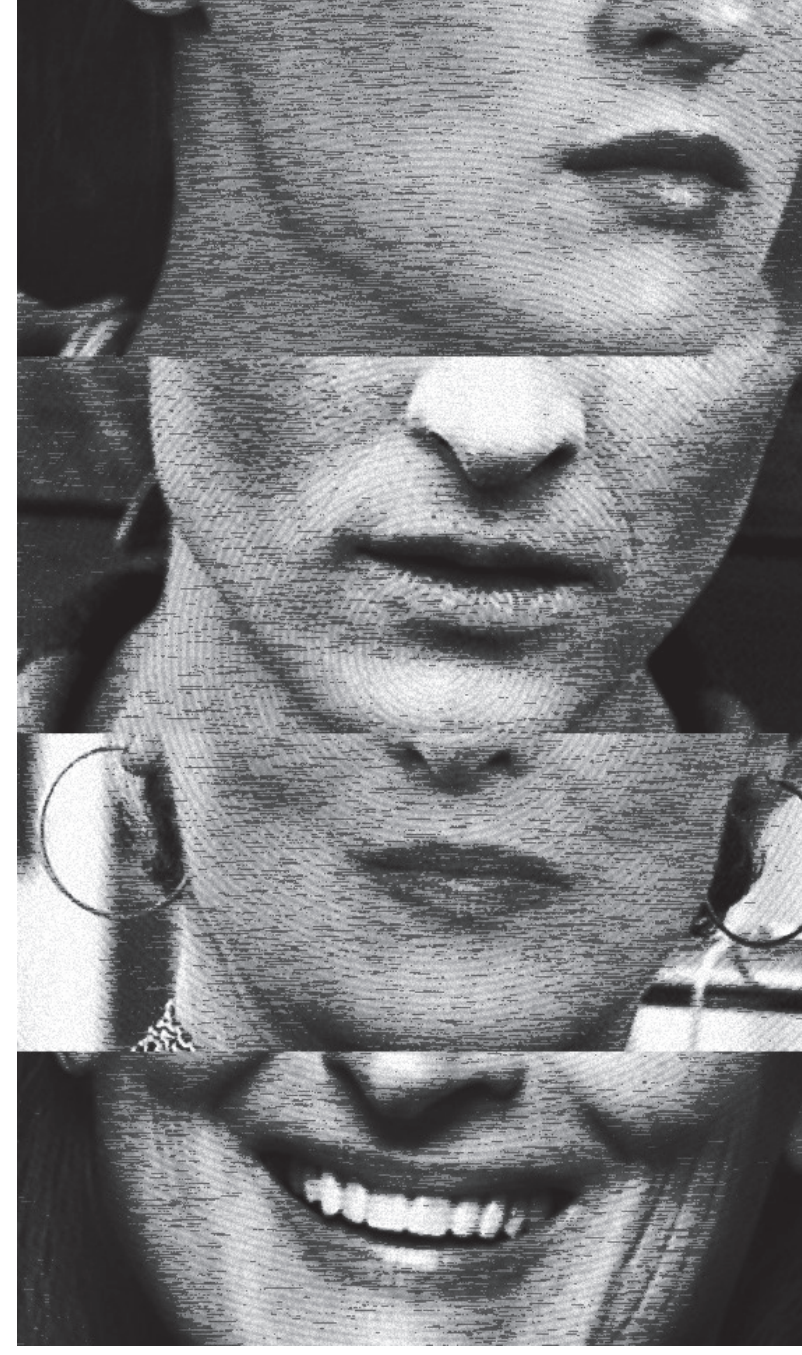


La situación a la que se hace referencia fue un momento ocurrido el 24 de noviembre de 2016, cuando Donald Trump se mofó de un periodista con una discapacidad congénita durante un acto político al imitar su modo de hablar diciendo, «Now, the poor guy — you ought to see the guy: 'Uh, I don't know what I said. I don't remember. Erm, maybe that's what I said'» ${ }^{10}$ (Good Morning America, 2017). Sin necesidad de nombrar a Trump ni al periodista involucrado, Streep puede dialogar con el político y exponerlo frente a la audiencia. En relación con las palabras de la actriz, la presentadora de noticias Robin Roberts expresó, «You know, it was quite a moment last night. Even though she never said his name, Trump's name, we knew exactly who she was talking about» ${ }^{11}$ (Good Morning America, 2017). Se comprueba de este modo la eficacia de la variante activa de discurso orientado utilizada por Streep.

Vale asimismo agregar que Streep utiliza otra estrategia adicional en sus referencias a Trump, «But there was one performance this year that stunned me. It sank its hooks in my heart. Not because it was good. There was nothing good about it» ${ }^{12}$ (citado en Izardi y Wand, 2017). La actriz presenta dos negaciones en su enunciado y con ellas describe a Trump como un personaje sádico. Si consideramos que «Se verá a menudo que la negación no-A sigue un desarrollo que, en virtud de ciertos principios de 'buen sentido' podría llegar a la conclusión A» (Ducrot, 1994, p. 139), podemos observar que, al indicar la Streep locutora del enunciado «Not because it was good. There was nothing good about it», convierte a Donald Trump en enunciador de «it was good. There was everything good about it", las afirmaciones opuestas. De este modo, puede subrayar la imagen de un Trump burlón y despiadado que se vuelve su interlocutor invisible durante gran parte de su discurso.

Meryl Streep puede resaltar de modo negativo la figura de Donald Trump sin siquiera nombrarlo, a la vez que lo mantiene como una presencia amenazante en todo momento. Durante el siguiente segmento de su discurso, sosteniendo su calidad de sujeto empírico de la enunciación, la actriz retiene su rol de locutora y advierte, «Disrespect invites disrespect. Violence incites violence» ${ }^{13}$ (citado en Izardi y Wand, 2017). En este caso, la locutora es responsable de la puesta en escena del punto de vista proverbial, si bien no es la locutora de los proverbios en sí. Se subraya una vez más el carácter político del discurso de la actriz para reprochar los agravios de Trump. Como indican García Negroni y Tordesillas Colado (2001), este tipo de apreciaciones «se aleja de esta manera de la propia subjetividad individual y adquiere un papel virtual que posibilita un alejamiento de la responsabilidad» (p. 176). Su discurso toma un carácter de universalidad y un tono pedagógico y admonitorio a través de las fuentes tópicas que utiliza, que llevan sus palabras a un nivel casi proverbial y se constituyen como ideas universales.

En la sección siguiente, Streep realiza un llamado a defender la prensa, si bien en sus breves palabras al respecto no se vale de discurso referido. 
Por último, en el séptimo segmento de su alocución de aceptación del Premio Cecil B. DeMille, la actriz vuelve su enunciación más personal y emotiva a través de dos instancias de discurso directo. En el discurso directo oral puede observarse «un cambio entonativo que indica el paso de un discurso a otro» (García y Tordesillas, 2001, p. 164). En ambos casos se anuncia el discurso directo al introducirlo con el mismo verbo de decir, «said to me» ${ }^{14}$. Como es usual en el discurso directo, «el discurso del locutor que cita presenta el discurso del locutor citado como la actualización fiel del discurso que el locutor citado pronunció en su momento» (García y Tordesillas, 2001, p. 165). Considero que, en casos como estos de presentaciones públicas de índole no legal, la fidelidad del discurso citado es a los ojos de los interlocutores más bien una convención, ya que no sería extraño que, por diversas razones (como lapsos de memoria, voluntad estética o fallidos del subconsciente), hubiera operado aquí - y operaran en general en casos similares - ciertas estilizaciones en lugar de una reproducción verbatim de la palabra.

Como se ha indicado, ambas instancias vuelven la enunciación más personal y conectada con el arte de la actuación, sin por ello perder la voluntad política que permeó todo el contenido del discurso. En primer término, Streep recuerda una anécdota en la que ella se quejaba de alguna banalidad como tener que perderse la cena por estar en el set de filmación y un colega le recuerda su compromiso con la profesión, «Tommy Lee Jones said to me, 'Isn't it such a privilege, Meryl, just to be an actor?' ${ }^{15}$ (citado en Izardi y Wand, 2017). Como explica Ducrot, «el locutor puede ceder el lugar de enunciador al alocutario o a un tercero y ocupar el del destinatario» (1994, p. 140). Utilizar las palabras de un veterano actor, director y productor con más de 35 años en la profesión, que ha sido galardonado con el Premio Oscar y el Globo de Oro, y que goza del reconocimiento de sus pares, es una estrategia que aquí hace que las palabras de Jones, como «discurso independiente con respecto al discurso del locutor que cita» (García y Tordesillas, 2001, p. 164), ratifiquen, apoyen y reafirmen la posición de Streep y le permite avanzar hacia el final de su discurso.

Las últimas palabras de Meryl Streep de la noche fueron otra cita que se advierte también como recurso de autoridad que, en este caso, permite «deducir una conclusión de ese argumento mismo sin necesidad de demostrar su verdad» (Ducrot, 1994, p. 140). En esta oportunidad se trató de una instancia de discurso directo de una personalidad muy querida y admirada por la comunidad de Hollywood que había fallecido unas semanas antes, la actriz Carrie Fisher, «As my friend the dear departed Princess Leia said to me once, 'Take your broken heart, make it into art' ${ }^{16}$ (citado en Izardi and Wand, 2017). De este modo, Meryl Streep cerró su discurso de agradecimiento por el premio recibido, volviendo al tema de lo mucho que se siente afectada por la situación actual de Estados Unidos. Sin embargo, una vez más, al tomar las palabras de una figura reconocida como apoyo y consejo, y compartirlas con el público presente y los televidentes de todo el mundo, su enunciado no perdió optimismo frente a la adversidad. 


\section{Conclusiones}

El análisis del discurso de esta alocución permite observar cómo, a través de diversas instancias de discurso directo, discurso indirecto y discurso indirecto libre, Meryl Streep organizó sus ideas no solo para conformar una unidad en su enunciación al presentar el tema de Hollywood, los extranjeros y la prensa, sino para encadenarla a otras instancias de la ceremonia de premios. Al tomar al principio de su discurso las palabras que Hugh Laurie había dado cuando recibió su premio y finalizar su presentación con el recuerdo de Carrie Fisher, quien en la ceremonia había sido recordada en un homenaje póstumo poco más de media hora antes, Streep constituyó una estructura que va más allá de sus propias palabras y que, podría proponerse, construyó una red de solidaridad con sus pares y un sentimiento de comunidad con los artistas de Hollywood, la prensa y los extranjeros. Esta red de empatía, que Streep defiende con sus palabras, es la que se fortalece a través de los discursos reproducidos y de las numerosas referencias a tantos de sus colegas, del mismo modo que discute la figura de Donald Trump, aunque no lo nombre explícitamente. El discurso de Streep fue poderoso no solo por sus palabras sino por todas las otras voces de las que la actriz se apropió, tal como lo hace en el cine, sobre el escenario de la entrega de premios.

\section{Anexo}

\section{Discurso de aceptación de Meryl Streep del Premio Cecil B. DeMille ${ }^{17}$}

Thank you very much. Thank you. Please sit down. Please sit down. Thank you. I love you all. You'll have to forgive me. I've lost my voice in screaming and lamentation this weekend. And I have lost my mind sometime earlier this year. So I have to read.

Thank you, Hollywood foreign press. Just to pick up on what Hugh Laurie said, you and all of us in this room, really, belong to the most vilified segments in American society right now. Think about it: Hollywood, foreigners and the press. But who are we, and what is Hollywood, anyway? It's just a bunch of people from other places. I was born and raised and educated in the public schools of New Jersey. Viola was born in a sharecropper's cabin in South Carolina, came up in Central Falls, Rhode Island. Sarah Paulson was born in Florida and raised by a single mom in Brooklyn.

Sarah Jessica Parker was one of seven or eight kids from Ohio. Amy Adams was born in Vicenza, Veneto, Italy, and Natalie Portman was born in Jerusalem — where are their birth certificates? And the beautiful Ruth Negga was born in Addis Ababa, Ethiopia, raised in Lon - in, no, in Ireland, I do believe, and she's here, nominated for playing a small-town girl from Virginia. Ryan Gosling, like all the nicest people, is Canadian. And Dev Patel was born in Kenya, raised in London, is here for playing an Indian raised in Tasmania. So Hollywood is crawling with outsiders and foreigners.

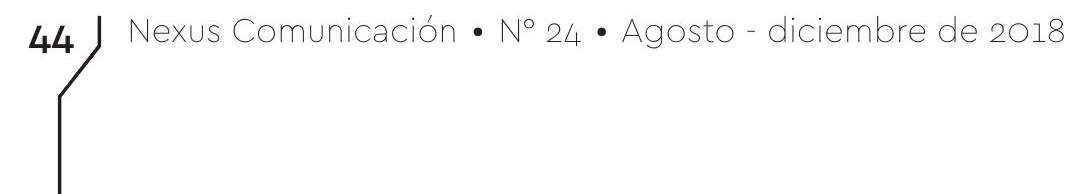


And if we kick them all out, you'll have nothing to watch but football and mixed martial arts, which are not the arts.

They gave me three seconds to say this, so. An actor's only job is to enter the lives of people who are different from us and let you feel what that feels like. And there were many, many, many powerful performances this year that did exactly that breathtaking, compassionate work. But there was one performance this year that stunned me. It sank its hooks in my heart. Not because it was good. There was nothing good about it. But it was effective and it did its job. It made its intended audience laugh and show their teeth. It was that moment when the person asking to sit in the most respected seat in our country imitated a disabled reporter. Someone he outranked in privilege, power and the capacity to fight back. It kind of broke my heart when I saw it. I still can't get it out of my head because it wasn't in a movie. It was real life.

And this instinct to humiliate, when it's modeled by someone in the public platform, by someone powerful, it filters down into everybody's life, because it kind of gives permission for other people to do the same thing.

Disrespect invites disrespect. Violence incites violence. And when the powerful use their position to bully others, we all lose. Okay. Go on with that thing.

Okay, this brings me to the press. We need the principled press to hold power to account, to call them on the carpet for every outrage. That's why our founders enshrined the press and its freedoms in our Constitution. So I only ask the famously well-heeled Hollywood foreign press and all of us in our community, to join me in supporting the Committee to Protect Journalists, because we're going to need them going forward, and they'll need us to safeguard the truth.

One more thing. Once when I was standing around on the set one day whining about something - you know, we were going to work through supper, or the long hours or whatever - Tommy Lee Jones said to me, «Isn't it such a privilege, Meryl, just to be an actor?»Yeah, it is. And we have to remind each other of the privilege and the responsibility of the act of empathy. We should all be very proud of the work Hollywood honors here tonight. As my friend the dear departed Princess Leia said to me once, «Take your broken heart, make it into art.» Thank you, foreign press. 
1 Traductor Público Nacional de Inglés (Univ. Nacional de Córdoba, Argentina) Bachelor of Arts English (Whitman College, EE UU). Profesor de Lengua y Literatura Inglesa (Univ. Nacional de Córdoba, Argentina). Magíster en Inglés - Orientación Literatura Angloamericana (Univ. Nacional de Córdoba, Argentina). Máster en Política y Gestión Universitaria (Universidad de Barcelona, España).

2 Perdí la voz gritando y lamentándome este fin de semana. Y perdí la cordura en algún momento de este año.

3 Para tomar las palabras de Hugh Laurie, ustedes y todos nosotros en este salón de hecho pertenecemos a los segmentos más vilipendiados de la sociedad estadounidense actual. Piénsenlo: «Hollywood, los extranjeros y la prensa».

${ }^{4}$ Gracias a la Asociación de la Prensa Extranjera de Hollywood por este asombroso honor. Supongo que lo que lo hace más asombroso es el hecho de que podré decir que lo gané en los últimos Globos de Oro de la historia [...] No quiero ser pesimista. Sucede que tiene las palabras «Hollywood», «extranjera»y «prensa» en el título. También creo que, para algunos republicanos, hasta la palabra «asociación» es un tanto sospechosa.

${ }^{5}$ Ustedes y todos nosotros en este salón de hecho pertenecemos a los segmentos más vilipendiados de la sociedad estadounidense actual.

${ }^{6}$ Pero ¿quiénes somos, y en todo caso qué es Hollywood? Es un montón de gente de otros lados.

${ }^{7}$ ¿Dónde están sus partidas de nacimiento?

8 Así que Hollywood está plagado de marginales y extranjeros, y si los echamos a todos a patadas, no van a tener nada para ver excepto fútbol americano y artes marciales mixtas, Y esas no son las Artes.

${ }^{9}$ Fue ese momento en el que la persona que pide ocupar el lugar más respetado de nuestro país imitó a un reportero discapacitado.

${ }^{10}$ Entonces el pobre tipo - deberían verlo: «Ehm, no sé qué dije. No recuerdo. Ehm, quizás eso es lo que dije».

11 Fue un momento fuerte anoche. Aunque nunca pronunció su nombre, el nombre de Trump, sabíamos con exactitud que se refería a él.

12 Pero hubo una actuación este año que me sobrecogió. Me clavó sus garfios en el corazón. Y no porque fue buena; no hubo nada de bueno en esa actuación.

13 La falta de respeto invita a la falta de respeto. La violencia engendra la violencia.

${ }^{14}$ Me dijo.

15 Tommy Lee Jones me dijo: «Meryl, qué privilegio es ser actor ¿no?»

16 Como una vez me dijo mi querida amiga, la ya fallecida Princesa Leia: «Toma tu corazón hecho pedazos y conviértelo en arte».

${ }^{17}$ (Izardi y Wand). 


\section{Referencias}

Ducrot, O. (1994). El decir y lo dicho. París: Hachette Universidad.

García, M. y Tordesillas, M. (2001). La enunciación en la lengua. De la deixis a la polifonía. Madrid: Gredos.

Good Morning America. «ABC News.»09 de 01 de 2017. YouTube. Web. 15 de 01 de 2017. <https: / / www. youtube.com/watch? $=$ YItOtHrYA-U>.

Izardi, E. y Wand, A. «Meryl Streep called out Donald Trump at the Golden Globes. He responded by calling her 'over-rated.'.» The Washington Post 09 de 01 de 2017. web. 15 de 01 de 2017. <https://www.washingtonpost.com/news/arts-and-entertainment/wp/2017/01/08/ meryl-streep-called-out-donald-trump-at-the-golden-globes-read-her-speech-here/?utm_ term $=$. fb9fc9252c1e $>$.

Keneally, M. Donald Trump's History of Raising Birther Questions About President Obama. 18 de 09 de 2015. Web. 15 de 01 de 2017. <http://abcnews.go.com/Politics/donald-trumps-historyraising-birther-questions-president-obama/story?id=33861832>.

THR Staff. «Golden Globes: Hugh Laurie Wins Best Performance by a Supporting Actor in TV.» The Hollywood Reporter 08 de 01 de 2017. Web. 08 de 01 de 2017. <http://www. hollywoodreporter.com/news/hugh-laurie-wins-best-performance-by-a-supportingactor-tv-golden-globes-2017-960456>.

Recibido: 17 de Abril de 2018 / Aprobado: 1 de diciembre de 2018
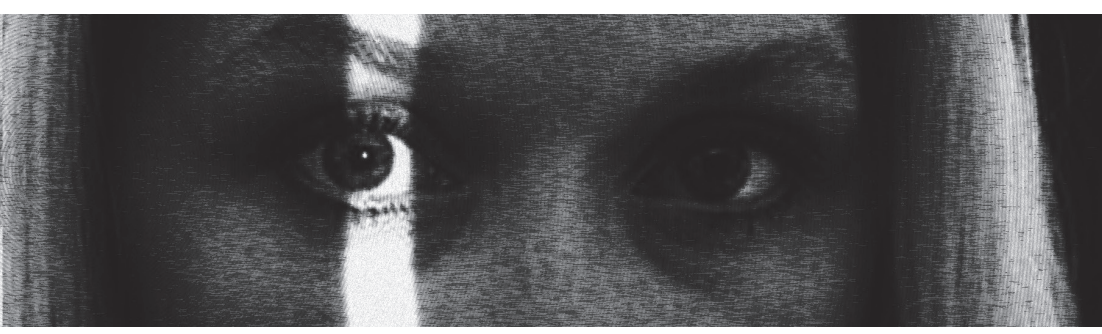